\title{
Improving the acoustic efficiency of laminated dissipative noise silencers for boiler gas-air paths
}

\author{
Evgeniy Zhuravlev ${ }^{1, *}$, Dmitry Chugunkov ${ }^{1}$, and Galina Seyfelmlyukova ${ }^{1}$ \\ ${ }^{1}$ National Research University "MPEI", Moscow, Russian Federation
}

\begin{abstract}
The operation of thermal power facilities is associated with the negative impact on the environment and humans of such factors as air, water, soil pollution, electromagnetic radiation, and noise. This article is devoted to improving the noise reduction efficiency of gas and air paths of boilers of thermal power plants. In world practice, to reduce noise in gas-air ducts, dissipative noise silencers are used. The authors propose a new design of sound attenuation elements for dissipative plate-type silencers. The article defines the acoustic and aerodynamic characteristics of noise silencers with new sound attenuation elements. We compare the characteristics of silencers made of new silencing elements and the characteristics of silencers consisting of plates of common design with flat surfaces of the side walls, and demonstrate an increase in acoustic efficiency and a decrease in pressure loss when using the proposed new silencing elements in noise silencers. Recommendations on the use of dissipative plate-type silencers with developed sound attenuation elements for gas-air paths of boilers are given.
\end{abstract}

\section{Introduction}

The annual increase in the consumption of heat and electric energy is associated with scientific and technological progress and an increase in the quality of life, as well as the emergence of new consumers, which include industrial enterprises and residential neighborhoods. To meet the increasing demand for energy, many energy objects are built, modernized and expanded [1].

We should note that energy has a significant negative impact on the environment. Sources of this effect are boiler houses, district heating stations of various sizes and, mainly, combined heat and power facilities (CHPs).

CHPs negatively affect the environment and humans through pollution of air (emissions of carbon dioxide, carbon monoxide and sulfur dioxide, nitrogen oxides and ash) and water basins, electromagnetic radiation and noise exposure.

The operation of the equipment of any energy facility is always associated with the emission of noise, which may exceed permissible norms [2].

At CHPs and boiler houses, the most powerful sources of environmental noise are the mouths of chimneys and the air intakes of blowers. The noise propagating through the gas paths and emitted from the mouths of the chimneys is generated as a result of the fuel combustion process in the boiler furnaces [3] and during the operation of draft machines (smoke exhausters and blower fans).
An effective method of reducing noise from gas-air paths of boilers is the use of noise silencers [4, 5, 6, 7].

There are three main types of silencers:

- reactive;

- active;

- dissipative (absorption).

A reactive silencer operates on the principle of multiple reflection and scattering of sound waves on acoustic filters, which are formed by a combination of acoustic mass and elasticity in the form of chambers and connected tubes [8-11]. Such designs are used in the gasair paths of small-section boilers, characterized by increased pressure losses; installed after the safety valves of power boilers and in the exhaust systems of internal combustion engines.

The active silencer works on the principle of superimposing acoustic waves in antiphase on the existing noise spectrum. The greatest decrease in such silencers is achieved at frequencies of $125 \div 250 \mathrm{~Hz}$ of geometric mean octave bands. They are used in gas-air paths of small sections and in non-dusty environments, have a high manufacturing cost, require complex adjustment and periodic technical inspections.

The dissipative silencer works on the principle of partial conversion of sound energy into thermal energy. A noise suppressor of this type, as a rule, consists of one or several sound attenuation elements, each of which has an outer shell of thin-walled metal with a section of a perforated surface [12-20]. The inner space of the element is filled with sound-absorbing material (SAM), placed in a shell of sound-permeable material, which

\footnotetext{
*orresponding author: ZhuravlevYA@mpei.ru
} 
protects the SAM from being blown out by the flow of gaseous medium from the space of the sound attenuation element.

The location of the sound attenuation elements and their geometric dimensions significantly affect the sound mitigation level and the pressure loss introduced by the silencer design.

Certain requirements are imposed on SAM: fire resistance, hydrophobicity, large surface area, low density. In the energy sector, various types of mineral wool are used as SAM: glass, stone (basalt) and slag; as well as polystyrene foam and polyurethane foam. The most widespread SAM filling of silencing elements for dissipative silencers was basalt mineral wool, which fits all of the above requirements.

Competitive advantages of dissipative silencers are broadband sound attenuation, simplicity of design, long service life and lack of operating costs.

Figure 1 shows the main types of absorption (dissipative) silencers.
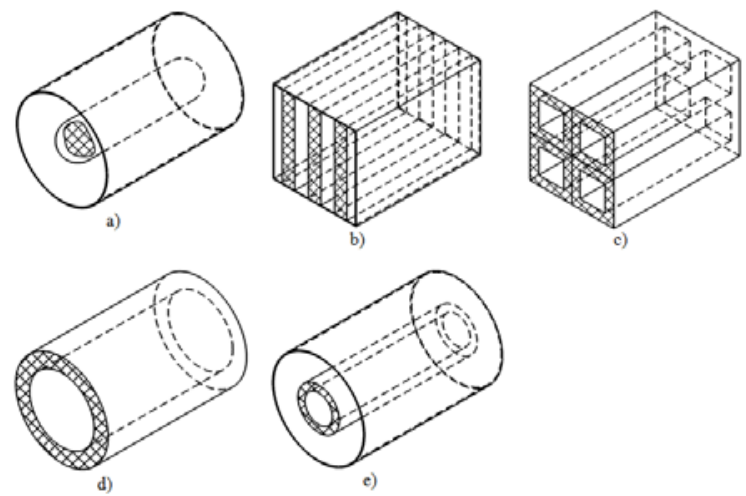

Fig.1. Types of dissipative silencers: a - rocker, b - laminated, c - cellular, d - cylindrical, e - tubular

\section{Methods}

With a decrease in noise in the gas-air paths of boilers, the most widespread was a laminated dissipative silencer (Fig. 2). A laminated silencer is a set of parallel plates that consist of noise attenuation elements (cassettes) of a rectangular cross section with a flat surface of the side perforated walls.

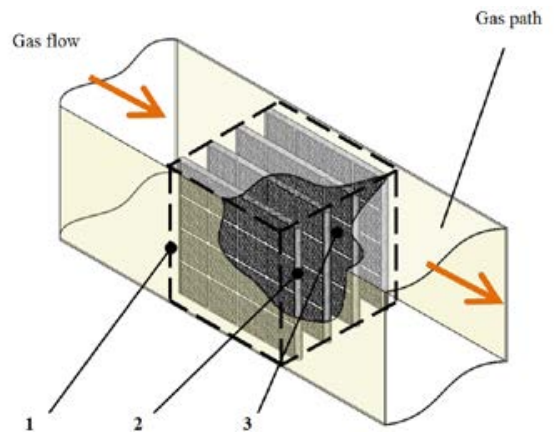

Fig.2. Dissipative laminated silencer in the air duct: 1 is a noise silencer, 2 is a plate, 3 is a sound attenuation element (cassette)

Installation of a silencer in the gas-air path of the boilers (channel) leads to reduction in its cross-section and to creation of additional hydraulic resistance.
A silencer in the gas-air path, providing the required acoustic efficiency, can introduce additional pressure losses, which will lead to a decrease in boiler performance. Therefore, when creating noise silencers with the required acoustic efficiency, it is important to minimize pressure losses.

Let us consider possible ways to improve laminated dissipative noise silencers in order to increase the sound attenuation efficiency and reduce the introduced pressure loss.

The main parameter of a silencer is its acoustic efficiency. Sound attenuation in a laminated silencer is determined by the formula by A.I. Belov [21], dB / m:

$$
\Delta L_{p \& \&}=4,4 \cdot \mathbb{Q S}^{\prime} \cdot \frac{\mathbb{1}}{D_{\text {foys }}}
$$

where $l$ is length of the silencer, $\mathrm{m}$; $D_{\text {hyd }}$ is hydraulic diameter of the silencer cross-section, $\mathrm{m}, \alpha^{\prime}$ is sound absorption coefficient

The hydraulic diameter of the silencer, $m$, is determined by:

$$
D_{\text {foyd }}=\frac{4 \cdot 5_{\mathbb{E} Q}}{P}
$$

where $P$ is wetted perimeter of the silencer, $\mathrm{m} ; S_{c s}$ is silencer cross-sectional area, $\mathrm{m}^{2}$

The relative flow area of the silencer in the channel, $\%$, is determined by:

$$
k_{0}=\frac{s_{c a}}{5} \cdot 100 \%
$$

where $S$ is area of the channel cross-section, $\mathrm{m}^{2} ; S_{c s}$ is silencer cross-sectional area, $\mathrm{m}^{2}$

The second important parameter of silencers is the pressure loss they introduce. The pressure loss of the laminated silencer is determined by the well-known formula [22], $\mathrm{Pa} / \mathrm{m}$ :

$$
\Delta p=\left[\xi_{\text {and }} \cdot\left(1-\frac{k_{0}}{100}\right)+\xi_{\text {out }} \cdot\left(1-\frac{k_{0}}{100}\right)^{2}+\left(\xi_{f y}-0.015\right) \cdot \frac{l}{D_{\text {hys }}}\right] \cdot \frac{9^{2}}{2}
$$

where $\xi_{\text {in }}$ and $\xi_{\text {out }}$ are input and output resistance coefficients; $\xi_{\text {fr }}$ is friction coefficient; $l$ is silencer length; $D_{\text {hoy }}$ is silencer hydraulic diameter, $\mathrm{m} ; \vartheta$ is medium flow velocity in the cross section of the silencer, $\mathrm{m} / \mathrm{s} ; k_{0}$ is silencer relative flow area, $\% ; \rho$ is medium flow density, $\mathrm{kg} / \mathrm{m}^{3}$

Analyzing formulas (1) and (4), we can conclude that the same parameters, on the one hand, increase the attenuation of sound in the silencer, and on the other hand, increase its hydraulic resistance.

When creating a silencer, it is necessary to ensure the maximum efficiency of the silencer when it forms pressure losses within acceptable limits. According to the formula (1), to increase the acoustic efficiency, the silencer's hydraulic diameter is minimized, which is achieved by the formula (2) by reducing the passage area of the silencer (the cassettes are very close to each other) and increasing its wetted perimeter (increasing the area of the sound-absorbing surfaces of the cassettes). The reduction in pressure loss according to the formula (4) is achieved by reducing the values of the input and output resistance coefficients by installing streamlined elements. 
In many cases, a problem arises when the standard design of the silencer cannot provide the required noise reduction while ensuring acceptable pressure losses, therefore, the task of improving the noise attenuation elements of dissipative laminated silencer $\mathrm{s}$ is relevant.

To increase the efficiency of the silencer and reduce its hydraulic diameter, we propose to use a series of noise silencer cassettes with developed lateral sound absorption surfaces (increased wetted perimeter). The largest sound absorption area of the side surfaces of the cassettes is achieved when using the profile of the side walls in the form of half-cylindrical concavities. Further studies will be carried out for cassettes with halfcylindrical concavities with equal diameters in the range $\mathrm{D}=10 \div 80 \mathrm{~mm}$ (IS cassette). Figure 3 shows the developed model of the IS cassette of the original design [23], designed for a laminated noise silencer.

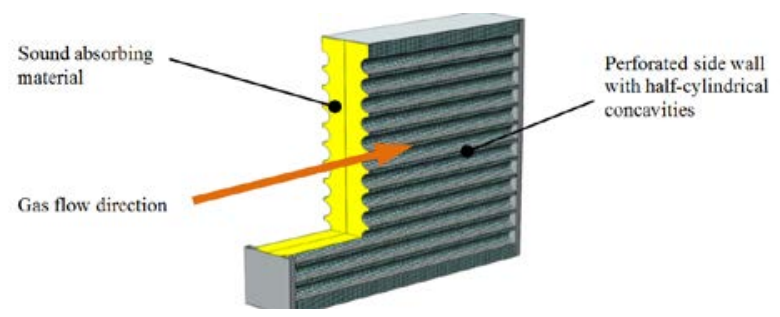

Fig.3. IS cassette for a laminated dissipative silencer

\section{Results and Discussion}

\subsection{Study of acoustic and aerodynamic characteristics of silencers made of new elements of sound attenuation}

When conducting studies to determine the characteristics of a noise silencer consisting of IS cassettes the influence of the speed of the medium in the channel on noise generation was not taken into account.

To demonstrate the superiority of the technical characteristics of the noise silencer, consisting of IS cassettes, in comparison with the noise silencer, consisting of standard cassettes with flat side walls (FS cassettes), we have conducted comparative theoretical studies.

We have assumed that the coefficient relative to the exhaust section of the silencers, the number of cassettes and their overall geometric dimensions are identical. And in order to maintain the evenness of the silencer cross section, made up of IS cassettes, when varying the diameters of half-cylindrical concavities $\mathrm{D}$, the width of channel B was decreasing (Figures 4a and 4b).

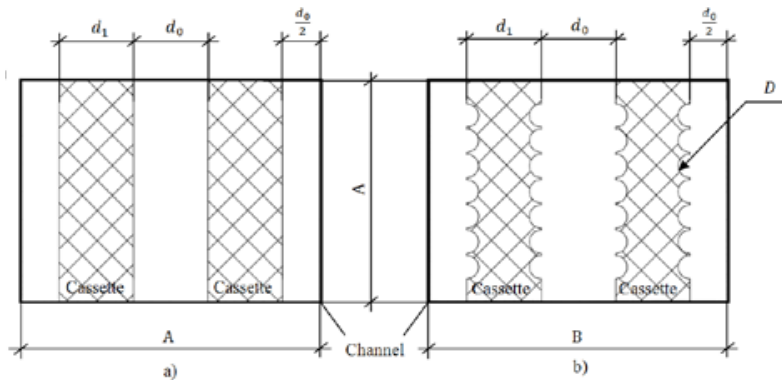

Fig.4. The layout of the silencer cassettes in the channel: a) a noise silencer made up of FS cassettes; b) a noise silencer made up of IS cassettes; $D$ is the diameter of the halfcylindrical concavity; A, B are channel length and width, respectively; $d_{1}$ is cassette thickness; $d_{0}$ is distance between cassettes

By the formulas (1) and (4), the sound attenuation and the introduced pressure loss were determined by the silencer from the PS cassettes and the silencer from the BC cassettes at $k_{0}=33 \%, k_{0}=50 \%$ and $k_{0}=67 \%$. Their comparison is done in Figure 5.
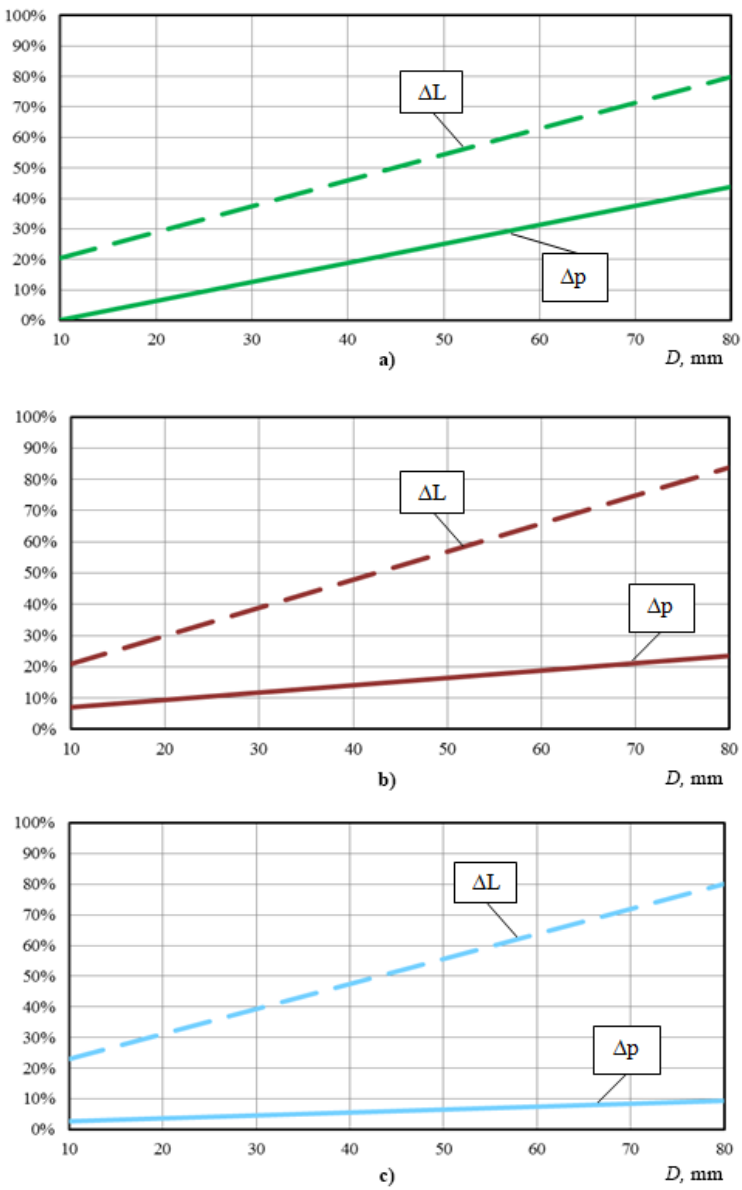

Fig.5. The change in the percentage of acoustic efficiency $\Delta \mathrm{L}$ and pressure loss $\Delta \mathrm{p}$ by a silencer from cassettes of aircraft with concavities of diameter $D$ compared to a silencer from cassettes of PS at $k_{0}=67 \%$ (a), $k_{0}=50 \%$ (b) and $k_{0}=33 \%$ (c)

From Figure 5, we can conclude that it is preferable to use laminated noise silencers from the developed IS cassettes, the half-cylindrical concavities of which have 
the largest diameter, and also to reduce the silencer cross-section (reduce the distance between the cassettes). The increase in acoustic efficiency with the use of IS cassettes is observed for all three relative cross-sections, and its values are comparable to each other and range from 20 to $84 \%$. The pressure loss increases with increasing diameter of the concavities and the relative flow area of the silencer. So, with $k_{0}=67 \%$ and a concavity diameter of $80 \mathrm{~mm}$, the pressure loss was $44 \%$ of the loss value created by a typical laminated silencer from cassettes with flat side walls.

\subsection{The effect of using new sound attenuation elements}

A typical laminated silencer consists of a certain number of cassettes and is installed in the gas path of the boiler. Acoustic and aerodynamic parameters can be determined using a known method [21]. Let us ask ourselves: what will change if in place of FS cassettes with flat side walls we install IS cassettes with concavities with similar overall dimensions? Obviously, the area of sound absorption and the cross section of the silencer will increase due to the concavities of the cassettes.

Figure 6 shows the acoustic, calculated according to (1), and aerodynamic, calculated according to (4), characteristics of two noise silencers: a silencer made of IS cassettes and a silencer made of FS cassettes, the differences between which were only in the type of cassettes in use. The initial values were: the geometrical dimensions of the gas path $4000 \times 4000 \mathrm{~mm}$, the number of cassettes - 112 pcs., the thickness of the cassettes $200 \mathrm{~mm}$, the distance between the cassettes - $187 \mathrm{~mm}$, and the distance between the outer cassettes and the wall of the path $-371 \mathrm{~mm}$. The diameter of the concavities of the IS cassettes was $60 \mathrm{~mm}$ with a step of $76 \mathrm{~mm}$. To reduce pressure losses, fairings were provided for the first and last silencer in the direction of the medium. The calculations were carried out for the most common medium flow velocity in the free path of $15 \mathrm{~m} / \mathrm{s}$. The sound absorption coefficients for the silencer made of IS cassettes in the first approximation, due to the lack of experimental data, were taken according to [21].

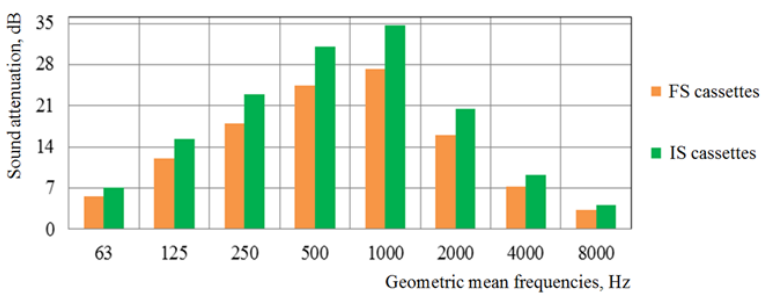

Fig.6. Sound attenuation by silencers

We can see from the Figure 6 that the silencer, consisting of IS cassettes, shows higher values of sound attenuation in comparison with the silencer, consisting of FS cassettes. This superiority in sound attenuation in all octave bands at geometric mean frequencies of 63 to $8000 \mathrm{~Hz}$ was 1.5 to $7.5 \mathrm{~dB}$.
According to the results of calculations using (3), it turned out that the use of IS cassettes can reduce pressure losses from $37 \mathrm{~Pa}$ (for FS cassettes) to $21 \mathrm{~Pa}$.

Additionally, a surface with semi-cylindrical concavities can be used as a lining of the gas-air duct to increase the total noise suppression efficiency [24].

The next stage in the study of original concave cassettes for a laminated dissipative noise silencer will be the creation of a prototype for determining the experimental values of sound attenuation, both in bench tests and on existing equipment, namely, after installing it in the gas paths of a boiler in some large CHP in Moscow.

\section{Conclusions}

1. On the basis of a typical cassette with a flat surface of the side walls of a plate-shaped dissipative noise suppressor, a new design of a sound attenuation element has been developed [23], the main difference of which is the original shape of the side perforated walls, which are surfaces with half-cylindrical concavities.

2. According to the results of theoretical studies, the noise silencer made of the developed cassettes showed higher values of sound attenuation per $1 \mathrm{~m}$ of the length of the silencer in comparison with typical cassettes with a constant passage section of the silencer.

3 . In order to ensure high values of sound attenuation in the gas-air paths of boilers, we recommend to use noise silencers consisting of the proposed IS cassettes with a maximum concavity diameter.

4. We demonstrate that it is possible to achieve such a configuration of silencing elements (IS cassettes) of the silencer, when, with increased acoustic efficiency, it is possible to reduce pressure losses.

5. Additionally, a sound-absorbing surface in the form of semi-cylindrical concavities can be used as a lining of the internal walls of gas-air paths of boilers.

\section{Acknowledgements}

The study was carried out as part of a research program supported by a scholarship from the President of the Russian Federation SP-1944.2019.1.

\section{References}

1. A.I. Abramov, Improving the environmental safety of CHPs: a textbook for universities M.: Publishing House of MPEI, 378 (2002)

2. N.I. Ivanov, Engineering acoustics. Theory and practice of noise control: a textbook University Book, 424 (2016)

3. V.I. Krasnov, Development of methods for reducing noise from gas paths during the modernization of hot-water boilers of the PTVM type in the surrounding area [Text]: dis. Cand. Tech. Sciences: 05.14.01: defended 19.04.2005: approved. 10/15/2005 / Krasnov Valery Ivanovich. Moscow, 139 (2005) 
4. A.M. Drokonov, A.E. Drokonov, Noise reduction in power plants. Fundamental and applied problems of engineering and technology 3, 65-75 (2014)

5. B.V. Tupov, Increasing safety of thermal and nuclear power stations energy equipment by reducing noise. International Conference on Problems of Thermal Physics and Power Engineering 4, 14-16 (2017)

6. V.B. Tupov, S.A. Semin, B.V. Tupov, A.A. Taratornin, D.A. Rozanov, Noise barriers for powerplant equipment. Power Technology and Engineering, 50(6), 649-652 (2017)

7. S.A. Semin, V.B. Tupov, Effective measures to reduce the noise from a CHPP and a boiler-house. Proc. 5Th All-Russia Int. Sci. Pract. Conf. on Noise and Vibration Protection, 268-275 (2015)

8. V.B. Tupov, A.A. Taratorin, New steam silencers developed at MPÉI. Power Technology and Engineering 49, 4, 1, 301-303 (2015)

9. A.B. Permyakov, M.E. Marchenko, Acoustic pollution reduction media during steam discharges into the atmosphere. News of the Academy of Industrial Ecology, M. 30-31 (1995)

10. M.E. Marchenko, Yu.E. Mishenin, A.B. Permyakov, The sound attenuation system of safety valves of thermal power plants with supercritical steam parameters. News of the Academy of Industrial Ecology, 1, 88-91 (1997)

11. V.B. Tupov, D.V. Chugunkov, Silencers on steam vents of power boilers. Thermal Power Engineering 8, 34-37 (2009)

12. B.V. Tupov, V.T. Medvedev, Multistage silencers of gas paths with improved technical and economic characteristics. Power plants 8, 31-32 (2014)

13. B.V. Tupov, V.T. Medvedev, Determining the lengths of multi-stage silencers with different plate thicknesses. Thermal Engineering, 3, 66-69 (2014)

14. S. Yadav, G.S. Sharma, Noise reduction techniques for FD \& ID fan in thermal power plant using chamber based silencer. International Journal of Mechanical and Production Engineering Research and Development 9, 3 409-420 (2019)

15. A.E. Gjinolli, J.E. Dorgan, E.F. Ray, Dynamic analysis and design methods for combustion turbine exhaust silencers employing acoustical baffles. American Society of Mechanical Engineers ASME 20, Pressure Vessels and Piping Conference, PVP, 8, (2018)

16. V.B. Tupov, B.V. Tupov, Noise abatement of forced-draft fans using absorptive silencers. Power Technology and Engineering 51, 341-345 (2017)

17. V.B. Tupov, B.V. Tupov, Noise reduction from air intakes of compressors and blower fans. Proceedings of the INTER-NOISE 2016 45th International Congress and Exposition on Noise Control Engineering: Towards a Quieter Future, 21, 1446-1452 (2016)

18. N.A. Zroichikov, V.B. Prokhorov, V.B. Tupov, A.M. Arkhipov, M.V. Fomenko, Possible ways of reducing the effect of thermal power facilities on the environment. Thermal Engineering (English translation of Teploenergetika) 62(2), 146-153 (2015)

19. V.B. Tupov, D.V. Chugunkov, S.A. Semin, Reduction of noise emitted from exhaust paths of gas turbine units equipped with heat recovery boilers. Thermal Engineering (English translation of Teploenergetika), 56(1), 26-30 (2009)

20. V.B. Tupov, B.V. Tupov, D.A. Rozanov, Influence of aerodynamic factors on silencers of exhaust fans. Proceedings of the INTER-NOISE 45th International Congress and Exposition on Noise Control Engineering: Towards a Quieter Future, 1453-1460 (2016)

21. F.E. Grigoryan, E.A. Pertsovsky. Calculation and design of silencers for power plants. L .: Energy, 120 (1980)

22. I. Ye. Idelchik, Handbook of hydraulic resistance. Ed. M.O. Steinberg. 3rd ed., Revised. and app. M .; Engineering, 672 (1992)

23. D.V. Chugunkov, G.A. Seifelmlyukova, E.A. Zhuravlev, K.S. Fomenko, A.D. Skurikhina, A.E. Bogdanova. Sound-absorbing cassette for silencers of gas-air ducts: Pat. 187890 Rus. Federation; National Research University "MPEI”, 11 (2019)

24. D.V. Chugunkov, G.A. Seifelmlyukova, E.A. Zhuravlev, K.S. Fomenko, A.D. Skurikhina, A.E. Bogdanova. Sound-absorbing cassette for silencers of gas-air ducts: Pat. 1888650 Rus. Federation; National Research University “MPEI”, 9 (2019) 